Deniz, 0., Sánchez-Sánchez, C., Jaafar, R., Kharche, N., Liang, L., Meunier, V., ... Ruffieux, P. (2018). Electronic characterization of silicon intercalated chevron graphene nanoribbons on $\mathrm{Au}(111)$. Chemical

Communications, 54(13), 1619-1622. https://doi.org/10.1039/C7cc08353J Journal Name

\title{
Electronic characterization of silicon intercalated chevron graphene nanoribbons on $\mathrm{Au}(111)$
}

Received 00th January 20xx, Accepted 00th January 20xx

DOI: $10.1039 / x 0 \times x 00000 x$
O.Deniz, ${ }^{a}$ C. Sánchez-Sánchez, ${ }^{a,+}$ R. Jaafar, ${ }^{a}$ N. Kharche, ${ }^{b}$ L. Liang, ${ }^{c}$ V. Meunier, ${ }^{b}$ X. Feng, ${ }^{d}$ K. Müllen, R. Fasel, ${ }^{a, f}$ P. Ruffieux ${ }^{a}$

www.rsc.org/

\begin{abstract}
Electronic and thermal properties of chevron-type graphene nanoribbons can be widely tuned, making them interesting candidates for electronic and thermoelectric applications. Here, we use post-growth silicon intercalation to unambiguously access nanoribbons' energy position of their electronic frontier states. These are otherwise obscured by substrate effects when investigated directly on the growth substrate. In agreement with first-principles calculations we find a band gap of $2.4 \mathrm{eV}$.
\end{abstract}

The challenge of further improving the performance to size ratio brings conventional semiconductor materials to their limit and is poised to open opportunities for alternatives. In this respect, graphene-based materials and heterostructures derived thereof have attracted considerable attention and are already widely integrated in applications requiring high electron mobility in ultimately thin layers ${ }^{1-3}$ or large specific areas for efficient energy storage. ${ }^{4}$ With respect to switching devices, however, the missing electronic band gap of graphene sets clear limits to wider applications. One of the most promising strategies for opening a band gap is electron confinement in quasi one-dimensional graphene nanoribbons (GNRs), for which sizable band gaps suitable for roomtemperature switching are predicted for GNR width below $3 \mathrm{~nm} .{ }^{5}$ Recent advances in the bottom-up fabrication of GNRs have proven that the required atomic precision in width and

\footnotetext{
a. Empa, Swiss Federal Laboratories for Materials Science and Technology, Überlandstrasse 129, CH-8600 Dübendorf, Switzerland.

b. Department of Physics, Applied Physics, and Astronomy, Rensselaer Polytechnic Institute, Troy, New York 12180, United States.

c. Center for Nanophase Materials Sciences, Oak Ridge National Laboratory, Oak Ridge, Tennessee 37831, USA

d. Chair of Molecular Functional Materials, Department of Chemistry and Food Chemistry, Technische Universität Dresden, Mommsenstrasse 4, D-01062 Dresden, Germany.

e. Max Planck Institute for Polymer Research, Ackermannweg 10, D-55128 Mainz, Germany.

f. Department of Chemistry and Biochemistry, University of Bern, Freiestrasse 3, $\mathrm{CH}$ 3012 Bern, Switzerland.

+ Currently at Instituto de Ciencia de Materiales de Madrid (ICMM-CSIC), Sor Juana Inés de la Cruz 3, 28049 Madrid, Spain.

Electronic Supplementary Information (ESI) available: Additional spectroscopy data of chevron GNRs adsorbed on $\mathrm{Au}(111)$ and on AuSIL. Wavefunction distribution of occupied and unoccupied states of chevron GNRs. See DOI: 10.1039/x0xx00000x
}

edge structure can indeed be achieved in a fully deterministic way by using specifically designed precursor monomers. These building blocks are selectively activated, polymerized and dehydrogenated on a metallic substrate surface to form the final GNR. ${ }^{6}$ Most recently, this strategy ${ }^{6}$ has been successfully expanded to GNRs of various width (and hence band gaps) ${ }^{7-13}$ and edge topologies. ${ }^{14-16}$

Conceptually, GNR properties can be further tuned by forming GNR heterostructures ${ }^{17,18}$ where a controlled alternation of chemical composition, width or edge topology would modulate band gap and edge-related electronic states along the GNR axis. This approach, however, requires the controlled addition of different precursor monomers in a predefined sequence, which has so far not been achieved. The class of chevron GNRs $^{6}$ bears significant potential to succeed along this path since an alternation of width, edge direction and chemical composition ${ }^{17,19-24}$ can be accomplished at the precursor monomer level and thus allows growth of homogenous GNRs with an unprecedented range of physical properties. ${ }^{25-29}$ For instance, defined replacement of carbon by nitrogen has been achieved, ${ }^{19-22}$ which allows modifying the energy alignment of the electronic states. ${ }^{17}$ In addition, theoretical simulations predict that the meandering geometry of chevron GNRs (Fig. 1a) results in intriguing thermoelectric properties since the phonon conductance can be altered almost independently from their electronic properties. ${ }^{30}$ Furthermore, a ferromagnetic ground state is predicted for chevron GNRs with zigzag edge segments ${ }^{26}$ with possible applications in spintronics relying on their tuneable magnetic properties while preserving the ballistic transport properties of straight GNRs. ${ }^{27}$

Despite the wide range of reported chevron GNR structures, an unambiguous experimental determination of their intrinsic band gap is still lacking. Here, we show that scanning tunnelling spectroscopy (STS) characterization of the as-grown chevron GNRs on the typically used $\mathrm{Au}(111)$ substrate is hampered by the overlapping contributions of the $\mathrm{Au}(111)$ surface state. This leads to a pronounced standing wave pattern along the meandering edges of the chevron 
GNRs. In order to overcome these limitations, we use in situ Si intercalation $^{31,32}$ to transform the $\mathrm{Au}(111)$ surface into an AuSi alloy (which we call AuSIL in the following) where the surface state is fully suppressed ${ }^{13}$ and hence allows to directly characterize the frontier orbitals by STS. On this basis, a band gap of $2.4 \mathrm{eV}$ is assigned to chevron GNRs, which is in excellent agreement with our first-principles calculations $(2.44 \mathrm{eV})$ taking into account band gap renormalization related to substrate screening effects.

Experiments were performed using a low-temperature scanning tunnelling microscopy system (LT-STM, ScientaOmicron) operated in the low $10^{-10} \mathrm{mbar}$ range. Samples were prepared in a connected preparation chamber. Repeated cycles of $\mathrm{Ar}^{+}$sputtering at $1 \mathrm{keV}$ and annealing at $745 \mathrm{~K}$ were applied for the preparation of a clean $\mathrm{Au}(111)$ surface, as judged by STM. Precursor molecules (6,11-dibromo-1,2,3,4tetraphenyltriphenylene, $\left.\mathrm{C}_{42} \mathrm{Br}_{2} \mathrm{H}_{26}\right)$ were sublimed from quartz crucibles (Kentax $\mathrm{GmbH}$ ) onto a clean $\mathrm{Au}(111)$ substrate held at $520 \mathrm{~K}$ for instant dehalogenation and polymerization. The polymers were then cyclodehydrogenated to form chevron GNRs by an annealing step at $710 \mathrm{~K}$. Finally, Si was evaporated from a Si bar $\left(20 \times 2 \times 0.5 \mathrm{~mm}^{3}\right)$ cut from a Si wafer $(\rho=1-8 \Omega . \mathrm{cm})$ by a home-made direct current evaporator onto the surface held at $475 \mathrm{~K}$. Due to substantial diffusion of $\mathrm{Si}$ into the Au bulk, the thickness of the AuSIL cannot be unambiguously determined. We therefore refer to the thickness needed to saturate the characteristic AuSIL surface ${ }^{13}$ as one monolayer. An electrochemically etched $W$ tip was used for STM and STS measurements. Prior to STS, the tip was conditioned by controlled indentation on AuSIL. Differential conductance $(\mathrm{d} / / \mathrm{d} V)$ spectra were taken by using a lock-in amplifier $\left(U_{r m s}=20 \mathrm{mV}, 860 \mathrm{~Hz}\right)$. Several spectra were recorded to accurately determine the valence band maximum (VBM) and conduction band minimum (CBM) of chevron GNR. The energy positions of the corresponding frontier states were determined differently depending on whether there is a wellresolved peak feature or a step-like spectral feature in the $\mathrm{d} / / \mathrm{d} V$ data: The peak maximum was taken in the former case, and the energy position at $80 \%$ of the step rise in the latter case, as motivated in Ref. ${ }^{33}$

DFT calculations were performed with the Vienna $A b$ initio Simulation Package (VASP) ${ }^{34,35}$ using the projector augmented wave (PAW) method $^{36}$ and the PBE exchange-correlation functional. $^{37}$ The atomic structure of the chevron GNR supported on the $A u(111)$ substrate was taken from earlier work. ${ }^{28}$ The polarizability $(\alpha)$ of the chevron GNR, used in the image-charge model, was calculated as follows. The longitudinal dielectric constant $(\varepsilon)$ of the nanoribbon was obtained using density functional perturbation theory (DFPT) ${ }^{38}$ as implemented in VASP. We employed a supercell size such that the distance between the periodic images was at least $15 \AA$, a plane-wave cut off of $400 \mathrm{eV}$ and a $16 \times 1 \times 1 k$-point grid. The polarizability of the chevron GNR was calculated using the 1D Clausius-Mossotti expression $\alpha=(A / 4 \pi)(\varepsilon-1)$, where $\mathrm{A}$ was the cross-sectional area of the supercell. The polarizability calculated by this method is $\alpha=34.30$. The distance between the chevron GNR and the image-plane position of the AuSIL
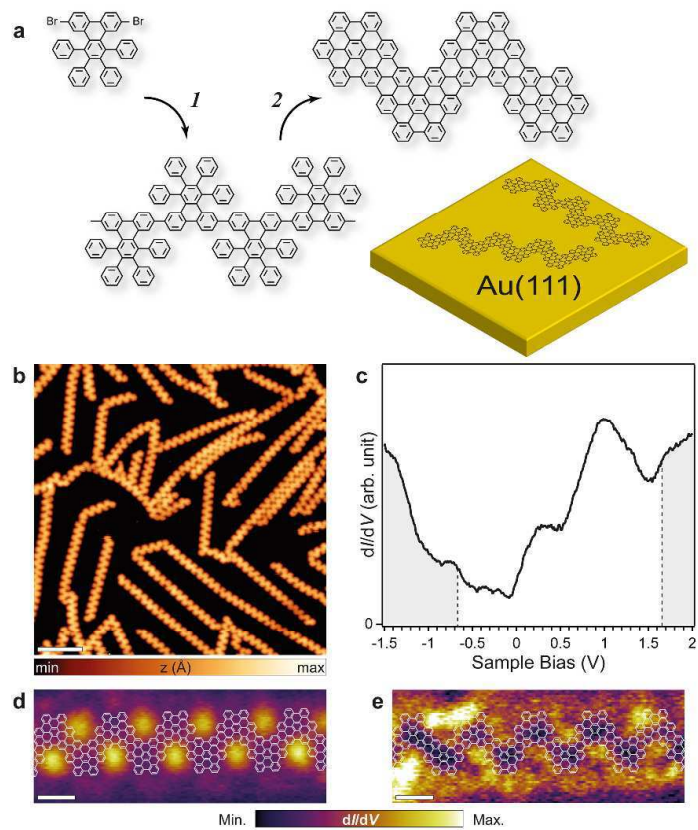

Min. dildV Max

Figure 1: Chevron GNR on $\mathrm{Au}(111)$ : (a) Reaction steps for the on-surface synthesis of chevron GNRs, (b) STM image (scale bar: $10 \mathrm{~nm}$, z-scale: $0.3 \AA-2.6 \AA ̊, U=-0.6 \mathrm{~V}, I=60$ $\mathrm{pA}, 5 \mathrm{~K}$ ) of chevron GNRs on $\mathrm{Au}(111)$, (c) averaged differential conductance spectrum $(U=-2 \mathrm{~V}, I=83 \mathrm{pA}, 77 \mathrm{~K})$ recorded on chevron GNR and differential conductance maps (scale bars: $1 \mathrm{~nm}, \mathrm{I}=83 \mathrm{pA}, 77 \mathrm{~K}$ ) recorded on chevron GNR segment at (d) $1 \mathrm{~V}$ and (e) at $1.85 \mathrm{~V}$.

substrate $(h)$ was taken to be $h=2.5 \AA . .^{13}$ Using these values of $\alpha$ and $h$, we calculated the band gap reduction $\left(\Delta E_{g, \text { model }}^{Q P}\right)$ due to the substrate screening within the image-charge model presented in Ref. ${ }^{39}$ Finally, we calculated the quasiparticle band gaps of the substrate-supported chevron GNR using $E_{\text {g,ads }}^{Q P}=E_{\text {g,iso }}^{G W-Q P}+\Delta E_{\text {g,model }}^{Q P}$, where $E_{\text {g,ads }}^{Q P}$ is the quasiparticle band gap of the substrate-supported chevron GNR and $E_{g, i s o}^{G W-Q P}$ was the GW quasiparticle band gap of a free-standing chevron GNR, which was taken from Ref. ${ }^{28}$

Chevron GNRs are fabricated on a Au(111) surface, following the scheme shown in Fig. 1a. Small-scale STM images (Fig. 1b) taken after polymerization and cyclodehydrogenation (Fig. 1a) confirm that the targeted chevron GNRs have been formed as described in the reaction scheme. Their electronic properties on $A u(111)$ are probed by $d / / d V$ spectra (Fig. 1c) and $d / / d V$ mapping (Fig. 1d,e). The spectrum in Fig. $1 \mathrm{c}$ is obtained by averaging several spectra recorded on a $1.6 \mathrm{~nm}$ wide grid across the GNR. The spectrum shows a small contribution of the $\mathrm{Au}(111)$ surface state onset at $\sim-0.4 \mathrm{~V}$. A second occupied feature $(-0.7 \mathrm{eV})$ is only resolved above the chevron GNRs and is assigned to the VBM. Its energy position is consistent with an earlier reported electron energy lossbased band gap determination of $\sim 2.8 \mathrm{eV}$, thereby assuming a similar work function and hence band alignment for chevron GNRs as for 7-AGNRs. ${ }^{40}$ For the unoccupied states where GNRrelated states overlap with the $A u(111)$ surface state, an assignment of the resolved features is much more difficult (see also Fig. S1 in Supplementary Information). We find main contributions at $0.3 \mathrm{eV}$ and $1.0 \mathrm{eV}$. $\mathrm{d} / / \mathrm{d} V$ mapping at these energies, however, reveals that their main contribution stems 

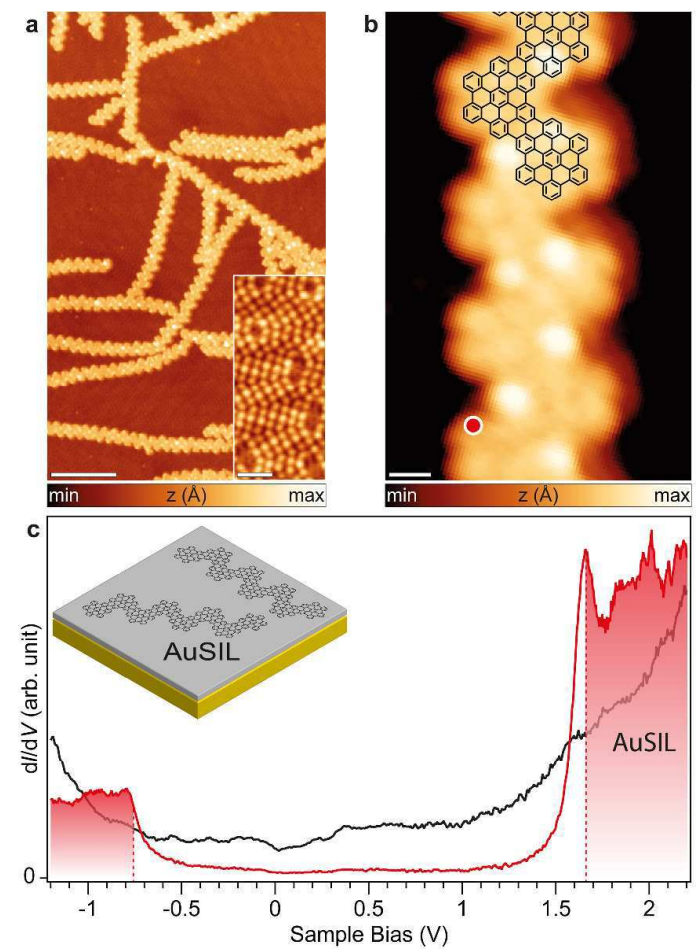

Figure 2: Si intercalated chevron GNRs: (a) overview STM image (scale bar: $10 \mathrm{~nm}, \mathrm{z}$ scale: $-1.3 \AA-3.7 \AA, U=-1.4 \mathrm{~V}, I=40 \mathrm{pA}, 5 \mathrm{~K}$ ) of chevron GNRs on AuSIL with an inset image (scale bar: $2 \mathrm{~nm}$, z-scale: $-0.3 \AA-0.3 \AA, U=1.7 \mathrm{~V}, I=200 \mathrm{pA}, 5 \mathrm{~K}$ ) showing the underlying AuSIL substrate, (b) high resolution STM image (scale bar: $0.5 \mathrm{~nm}$, z-scale: $0.2 \AA-2.6 \AA, U=-0.45 \mathrm{~V}, I=200 \mathrm{pA}, 5 \mathrm{~K}$ ) of a chevron GNR segment and (c) differential conductance spectra $(U=-1.2 \mathrm{~V}, I=500 \mathrm{pA}, 5 \mathrm{~K})$ on chevron GNR at the red dot in (b) and on AUSIL.

from the bays defined by the chevron GNRs and thus has to be assigned to a pronounced standing wave pattern of the $\mathrm{Au}(111)$ surface state (Fig. 1d). This interpretation is compatible with recently reported empty state STM images of chevron GNR on $\mathrm{Au}(111) .{ }^{41}$ Only the third unoccupied feature at $1.6 \mathrm{eV}$ shows main contributions close to the meandering GNR edge (Fig. 1e) and could therefore originate from the CBM of chevron GNRs. However, an unambiguous assignment on the basis of $d / / d V$ spectra of metal-adsorbed chevron GNRs is not possible.

In order to suppress the prominent standing wave pattern originating from the $\mathrm{Au}(111)$ surface state we formed an AuSIL layer between the GNRs and the Au substrate by in situ intercalation of Si with the sample held at $475 \mathrm{~K}$. We find that $\mathrm{Si}$ intercalation is similarly efficient as in the case of armchair GNRs (AGNRs). ${ }^{13}$ Fig. 2a demonstrates that a monolayer of AuSIL is intercalated under the chevron GNRs and that a new characteristic surface superstructure is formed. Compared to AGNRs, the edges of chevron GNRs are decorated with a somewhat higher density of $\mathrm{Si}$ adatoms, which is most prominent in the bays of the ribbon (Fig. 2b). Although the AuSIL layer is still metallic, the surface state is fully suppressed, resulting in an essentially featureless $d / / d V$ spectrum in the energy range where GNR-related states are expected (Fig. 2c). On the other hand, spectra taken above chevron GNRs now reveal prominent contributions at $-0.8 \mathrm{eV}$ for the occupied states and at $1.6 \mathrm{eV}$ for the unoccupied states, yielding a band gap of $2.4 \pm 0.1 \mathrm{eV}$. We find that the presence of Si atoms close to the edges does not impact the local electronic properties of chevron GNRs. Independent of the distance from Si adatoms we find the same band gap, and only the intensity of the frontier states is modified due to a different apparent height in the vicinity of adsorbed Si (see Fig. S2 in Supplementary Information). Also, the high amount of adsorbed Si does not modify the overall electronic structure of GNRs. Namely, the VBM and CBM positions indicate a similar alignment with respect to the vacuum level as observed for armchair GNRs with a significantly lower amount of adsorbed $\mathrm{Si}^{13}$

The quasiparticle band gap of the free-standing chevron GNR calculated using the GW approximation in our earlier work ${ }^{28}$ is $E_{g, i s o}^{G W-Q P}=3.62 \mathrm{eV}$. An independent work ${ }^{29}$ reported a band gap of $3.71 \mathrm{eV}$, in reasonable agreement with our earlier results. Both values are significantly larger than the experimentally measured band gap of $2.4 \mathrm{eV}$. This discrepancy is attributed to the fact that the GW calculations are performed on an isolated chevron GNRs while the experiments are performed on chevron GNRs supported on the AuSIL substrate where significant polarization effects are expected, especially in low-dimensional structures. It is now well established that the frontier orbital separation / band gap of low-dimensional nanostructures such as molecules ${ }^{42}$ or GNRs ${ }^{39}$ is strongly renormalized to a smaller value in the presence of an underlying substrate. This band gap reduction is attributed to the non-local screening of electron-electron interactions by the substrate, which leads to narrowing of the band gap. We use the image-charge model developed in Ref. ${ }^{39}$ to account for the substrate screening effects. To reduce the computational requirements, we model the chevron GNR as a uniform width ribbon with the effective width determined by the planar averaged wavefunctions shown in Fig. S3 in the Supplementary Information. Using this model, we obtain a band gap reduction induced by substrate screening of $\left|\Delta E_{g, \text { model }}^{Q P}\right|=1.18 \mathrm{eV}$. The quasiparticle band gap of the substrate-supported chevron GNR thus comes out to be $2.44 \mathrm{eV}$, in excellent agreement with the experimental value of $2.4 \mathrm{eV}$. Furthermore, we also apply the same image-charge model to Au-supported chevron GNRs in order to determine the CBM in this situation. The most important change in this configuration (GNR on $\mathrm{Au}(111)$ ) compared to the $\mathrm{Si}$ intercalated GNR/AuSIL/Au(111) system is the value of $h$ that is now $1.71 \AA^{28,39}$ It is known that adsorption height directly influences the screening imposed by the substrate. ${ }^{39}$ In this respect, our calculations reveal that the substrate screening renormalizes the GW band gap ${ }^{28}(3.62 \mathrm{eV})$ down to $2.26 \mathrm{eV}$. We have therefore firm evidence that the small peak at $1.6 \mathrm{eV}$ in the $\mathrm{d} / / \mathrm{d} V$ spectrum of the chevron GNRs on $A u(111)$ (Fig. 1c) is indeed the spectral feature of the CBM. The corresponding experimental band gap of $2.3 \mathrm{eV} \pm$ $0.1 \mathrm{eV}$ is in excellent agreement with the screened quasiparticle band gap of $2.26 \mathrm{eV}$ and a recent experimental investigation reporting a band gap of $2.5 \mathrm{eV}^{43}$

In conclusion, we have provided the first unambiguous spatially resolved spectroscopic data on chevron GNRs owing to the suppression of $A u(111)$ surface state through surface alloying at the chevron GNR/Au(111) interface. Si intercalation 
appears to be equally efficient for chevron GNRs as it has been reported for AGNRs. The results presented here highlight the significance of $\mathrm{Si}$ intercalation for various topologies of GNRs fabricated on $\mathrm{Au}(111)$, and hence identify $\mathrm{Si}$ intercalation as an efficient in situ surface modification to access the intrinsic electronic properties of the rapidly growing class of surfacesynthesized nanomaterials.

\section{Acknowledgement}

This work has been supported by the Swiss National Science Foundation, the Office of Naval Research BRC program (award N00014-12-1-1009), and the European Commission Graphene Flagship (No. CNECT-ICT-604391). CSS is grateful to Ministerio de Economía y Competitividad for financial support via the Juan de la Cierva Incorporación grant (IJCI-2014-19291, cofunded by the European Investment Bank). LL was supported by Eugene Wigner Fellowship at Oak Ridge National Laboratory.

\section{References}

1 P. Avouris, Nano Lett., 2010, 10, 4285-4294.

2 A. K. Geim and K. S. Novoselov, Nat. Mater., 2007, 6, 183-191.

3 K. S. Novoselov, A. K. Geim, S. V. Morozov, D. Jiang, M. I. Katsnelson, I. V. Grigorieva, S. V. Dubonos and A. A. Firsov, Nature, 2005, 438, 197-200.

4 M. F. El-Kady and R. B. Kaner, Nat. Commun., 2013, 4, 1475.

5 L. Yang, C.-H. Park, Y.-W. Son, M. L. Cohen and S. G. Louie, Phys. Rev. Lett., 2007, 99, 186801.

6 J. Cai, P. Ruffieux, R. Jaafar, M. Bieri, T. Braun, S. Blankenburg, M. Muoth, A. P. Seitsonen, M. Saleh, X. Feng, K. Müllen and R. Fasel, Nature, 2010, 466, 470-473.

7 L. Talirz, H. Söde, T. Dumslaff, S. Wang, J. R. Sanchez-Valencia, J. Liu, P. Shinde, C. A. Pignedoli, L. Liang, V. Meunier, N. C. Plumb, M. Shi, X. Feng, A. Narita, K. Müllen, R. Fasel and P. Ruffieux, ACS Nano, , DOI:10.1021/acsnano.6b06405.

8 Y.-C. Chen, D. G. de Oteyza, Z. Pedramrazi, C. Chen, F. R. Fischer and M. F. Crommie, ACS Nano, 2013, 7, 6123-6128.

9 A. Kimouche, M. M. Ervasti, R. Drost, S. Halonen, A. Harju, P. M. Joensuu, J. Sainio and P. Liljeroth, Nat. Commun., 2015, 6, 10177.

10 H. Zhang, H. Lin, K. Sun, L. Chen, Y. Zagranyarski, N. Aghdassi, S. Duhm, Q. Li, D. Zhong, Y. Li, K. Müllen, H. Fuchs and L. Chi, J. Am. Chem. Soc., 2015, 137, 4022-4025.

11 H. Huang, D. Wei, J. Sun, S. L. Wong, Y. P. Feng, A. H. C. Neto and A. T. S. Wee, Sci. Rep., 2012, 2, 983.

12 L. Talirz, P. Ruffieux and R. Fasel, Adv. Mater., 2016, 28, 62226231.

13 O. Deniz, C. Sánchez-Sánchez, T. Dumslaff, X. Feng, A. Narita, K. Müllen, N. Kharche, V. Meunier, R. Fasel and P. Ruffieux, Nano Lett., 2017, 17, 2197-2203.

14 P. Ruffieux, S. Wang, B. Yang, C. Sánchez-Sánchez, J. Liu, T. Dienel, L. Talirz, P. Shinde, C. A. Pignedoli, D. Passerone, T. Dumslaff, X. Feng, K. Müllen and R. Fasel, Nature, 2016, 531, 489-492.

15 J. Liu, B.-W. Li, Y.-Z. Tan, A. Giannakopoulos, C. SanchezSanchez, D. Beljonne, P. Ruffieux, R. Fasel, X. Feng and K. Müllen, J. Am. Chem. Soc., 2015, 137, 6097-6103.

16 D. G. de Oteyza, A. García-Lekue, M. Vilas-Varela, N. MerinoDíez, E. Carbonell-Sanromà, M. Corso, G. Vasseur, C. Rogero, E.
Guitián, J. I. Pascual, J. E. Ortega, Y. Wakayama and D. Peña, ACS Nano, 2016, 10, 9000-9008.

17 J. Cai, C. A. Pignedoli, L. Talirz, P. Ruffieux, H. Söde, L. Liang, V. Meunier, R. Berger, R. Li, X. Feng, K. Müllen and R. Fasel, Nat. Nanotechnol., 2014, 9, 896-900.

18 Y.-C. Chen, T. Cao, C. Chen, Z. Pedramrazi, D. Haberer, D. G. de Oteyza, F. R. Fischer, S. G. Louie and M. F. Crommie, Nat. Nanotechnol., 2015, 10, 156-160.

19 T. Marangoni, D. Haberer, D. J. Rizzo, R. R. Cloke and F. R. Fischer, Chem. - Eur. J., , DOI:10.1002/chem.201603497.

20 C. Bronner, S. Stremlau, M. Gille, F. Brauße, A. Haase, S. Hecht and P. Tegeder, Angew. Chem. Int. Ed., 2013, 52, 4422-4425.

21 C. Bronner, A. Haase and P. Tegeder, Phys. Rev. B, 2015, 91, 045428.

22 Y. Zhang, Y. Zhang, G. Li, J. Lu, X. Lin, S. Du, R. Berger, X. Feng, K. Müllen and H.-J. Gao, Appl. Phys. Lett., 2014, 105, 023101.

23 J. D. Teeter, P. S. Costa, M. M. Pour, D. P. Miller, E. Zurek, A. Enders and A. Sinitskii, Chem. Commun., 2017, 53, 8463-8466.

24 A. Radocea, T. Sun, T. H. Vo, A. Sinitskii, N. R. Aluru and J. W. Lyding, Nano Lett., 2017, 17, 170-178.

25 E. Costa Girão, E. Cruz-Silva, L. Liang, A. G. S. Filho and V. Meunier, Phys. Rev. B, 2012, 85, 235431.

26 E. Costa Girão, L. Liang, E. Cruz-Silva, A. G. S. Filho and V. Meunier, Phys. Rev. Lett., 2011, 107, 135501.

27 E. C. Girão, E. Cruz-Silva and V. Meunier, ACS Nano, 2012, 6, 6483-6491.

28 L. Liang and V. Meunier, Phys. Rev. B, 2012, 86, 195404.

29 S. Wang and J. Wang, J. Phys. Chem. C, 2012, 116, 10193-10197.

30 L. Liang, E. Cruz-Silva, E. C. Girão and V. Meunier, Phys. Rev. B, 2012, 86, 115438.

31 J. Mao, L. Huang, Y. Pan, M. Gao, J. He, H. Zhou, H. Guo, Y. Tian, Q. Zou, L. Zhang, H. Zhang, Y. Wang, S. Du, X. Zhou, A. H. C. Neto and H.-J. Gao, Appl. Phys. Lett., 2012, 100, 093101.

32 S. Lizzit, R. Larciprete, P. Lacovig, M. Dalmiglio, F. Orlando, A. Baraldi, L. Gammelgaard, L. Barreto, M. Bianchi, E. Perkins and P. Hofmann, Nano Lett., 2012, 12, 4503-4507.

33 H. Söde, L. Talirz, O. Gröning, C. A. Pignedoli, R. Berger, X. Feng, K. Müllen, R. Fasel and P. Ruffieux, Phys. Rev. B, 2015, 91, 045429.

34 G. Kresse and J. Furthmüller, Phys. Rev. B, 1996, 54, 11169.

35 G. Kresse and D. Joubert, Phys. Rev. B, 1999, 59, 1758.

36 P. E. Blöchl, Phys. Rev. B, 1994, 50, 17953-17979.

37 J. P. Perdew, K. Burke and M. Ernzerhof, Phys. Rev. Lett., 1996, 77, 3865-3868.

38 M. Gajdoš, K. Hummer, G. Kresse, J. Furthmüller and F. Bechstedt, Phys. Rev. B, 2006, 73, 045112.

39 N. Kharche and V. Meunier, J. Phys. Chem. Lett., 2016, 7, 15261533.

40 P. Ruffieux, J. Cai, N. C. Plumb, L. Patthey, D. Prezzi, A. Ferretti, E. Molinari, X. Feng, K. Müllen, C. A. Pignedoli and R. Fasel, ACS Nano, 2012, 6, 6930-6935.

41 T. H. Vo, U. G. E. Perera, M. Shekhirev, M. Mehdi Pour, D. A. Kunkel, H. Lu, A. Gruverman, E. Sutter, M. Cotlet, D. Nykypanchuk, P. Zahl, A. Enders, A. Sinitskii and P. Sutter, Nano Lett., 2015, 15, 5770-5777.

42 J. B. Neaton, M. S. Hybertsen and S. G. Louie, Phys. Rev. Lett., 2006, 97, 216405.

43 G. D. Nguyen, H.-Z. Tsai, A. A. Omrani, T. Marangoni, M. Wu, D. J. Rizzo, G. F. Rodgers, R. R. Cloke, R. A. Durr, Y. Sakai, F. Liou, A. S. Aikawa, J. R. Chelikowsky, S. G. Louie, F. R. Fischer and M. F. Crommie, Nat. Nanotechnol., , DOI:10.1038/nnano.2017.155. 